Wojtak, W., Ferreira, F., Erlhagen, W., \& Bicho, E.,

Learning Joint Representations for Order and Timing of Perceptual-Motor Sequences: a Dynamic Neural Field Approach

In: Proceedings of 2015 IEEE International Joint Conference on Neural Network (IJCNN 2015), pp. 1-7, Killarney, Ireland, July 12 - 17, 2015, DOI: 10.1109/IJCNN.2015.7280717 


\title{
Learning joint representations for order and timing of perceptual-motor sequences: a dynamic neural field approach
}

\author{
Weronika Wojtak* $^{*}$, Flora Ferreira ${ }^{\dagger}$, Wolfram Erlhagen ${ }^{\dagger} \S$ and Estela Bicho* \\ ${ }^{*}$ Research Center Algoritmi, University of Minho, Portugal \\ ${ }^{\dagger}$ Research Center for Mathematics, University of Minho, Portugal

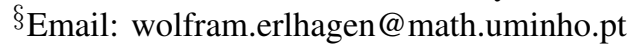

\begin{abstract}
Many of our everyday tasks require the control of the serial order and the timing of component actions. Using the dynamic neural field (DNF) framework, we address the learning of representations that support the performance of precisely time action sequences. In continuation of previous modeling work and robotics implementations, we ask specifically the question how feedback about executed actions might be used by the learning system to fine tune a joint memory representation of the ordinal and the temporal structure which has been initially acquired by observation. The perceptual memory is represented by a self-stabilized, multi-bump activity pattern of neurons encoding instances of a sensory event (e.g., color, position or pitch) which guides sequence learning. The strength of the population representation of each event is a function of elapsed time since sequence onset. We propose and test in simulations a simple learning rule that detects a mismatch between the expected and realized timing of events and adapts the activation strengths in order to compensate for the movement time needed to achieve the desired effect. The simulation results show that the effector-specific memory representation can be robustly recalled. We discuss the impact of the fast, activationbased learning that the DNF framework provides for robotics applications.
\end{abstract}

\section{INTRODUCTION}

Fluent execution of many of our everyday sequential activities requires the integration of information about both the temporal order and the timing of component actions. This is most obvious in skills like sports and music where a series of precisely timed movements has to be executed to guarantee a successful performance. But also in other routine tasks like for instance handing over an object to another person, the timing of the reach, grasp and place actions is fundamental for the smoothness and skillfulness of the joint behavior. The learning mechanisms that make such temporally extended behaviors possible are a matter of debate. On the basis of the observed tight coupling between the temporal and ordinal dimensions of serial behavior, it has been argued that a single learning system would be responsible for forming a fully integrated representation for sequencing and timing [6], [12], [17]. On this view, memory for the timing of events would also define the sequential event structure and vice versa. However, several experimental findings have been interpreted as evidence that the timing information may be stored independently of the order of actions it was combined with during training [21]. For instance, learning studies report a two stage learning process in which the acquisition of the ordinal sequence is typically much faster than the acquisition of the temporal sequence [15]. Moreover, once learned with a certain timing, the ordinal sequence of movements can be expressed with different movement rates, preserving the relative but not the absolute timing of component actions. Significant changes in the temporal pattern of the sequence may thus occur without affecting the ordinal structure.

In previous work we have developed a Dynamic Neural Field (DNF) model [10], [11] of sequence learning with a twofold goal in mind: 1) to address the open experimental question to which extent temporal and ordinal learning are independent processes, and 2) to use the insights from the modeling work to endow autonomous robots with the capacity to efficiently learn order and timing of perceptual-motor sequences.

The aim of the present paper is to extend our previous work by specifically addressing the important role of sensory feedback about executed actions for the learning process. Dynamic Neural Fields [2], [8] provide a rigorous mathematical framework to explain the formation of self-sustained activity patterns in neural populations which are the basis of the fast, activation-based learning implemented in the model. A memory layer represents the serial order and relative timing of events as an adjustable activation gradient over neural populations encoding instances of a cue (e.g., color, position or pitch) which guides sequence learning. The strength of activation of each event is a function of elapsed time since sequence onset. The serial order can be recalled with the correct timing by integrating in a decision layer the tonic input from the memory layer with a release of proactive inhibition [22] controlling the time to movement onset [10]. We have tested the DNF model in a real-world experiment in which the humanoid robot ARoS first memorizes a short musical sequence played by a human by watching color coded keys on a screen and then tries to execute the sequence on a keyboard from memory without external cues. ARoS was able to learn and represent in the memory gradient first the serial order and subsequently the precise timing of observed events in very few demonstration-execution cycles [11]. However, the correct sequence recall from a perceptually acquired memory was only possible since the fingers of the robot hand were positioned directed over the keys. ARoS could thus execute the keystrokes at the expected time with negligible (and constant) motor delays. Here, we 
present an extension of the DNF model that is able to learn effector-specific sequence representations that take into account varying movement times of component actions by integrating sensory and/or proprioceptive feedback. Evidence from neurophysiological studies suggest that abstract and motor-related representations of order and timing exist in separate but closely interacting neural structures [19]. Consisting with this hypothesis, the model implements the idea that the detection of a mismatch between the expected timing of the memorized perceptual event and executed timing of the same event drives the adjustment of the memory strength of the respective motor population. We show results of the learning and adaptation process in model simulations and discuss the impact of the findings for future robotics implementations.

\section{MODEL DESCRIPTION}

An overview of the model architecture consisting of several coupled dynamic neural fields is presented in the Fig. 1. The three fields on top implement the learning and memory of perceived sequential events. The five fields on bottom implement a novel motor-related learning mechanism that allows the cognitive system to adapt the order and timing of actions based on sensory feedback about the executed actions. This functionality of the perceptual sequence memory has been already tested in real-world experiments in which a human teacher demonstrates a sequence to the robot and the robot tries to recall this sequence from memory without any external cues. Several demonstration-execution cycles are usually necessary to ensure that the stored information is correct. Although different sources of sensory input may have been used as well (e.g., auditory) we refer in the following to the input from the vision system to have a concrete example in mind. In the robotics experiments, sequence learning was guided by a color cue, the fields are thus spanned over the dimension color.

The vision system provides the sensory information that is represented by neural populations in the perception field, $u_{P E R}$, which in turn triggers through excitatory connections (solid line) the evolution of localized activity pattern of corresponding populations in the perceptual sequence memory field, $u_{M E M}$. Inhibitory connections (dashed line) from $u_{M E M}$ to $u_{P E R}$ destabilize the bump in the perception field, which ensures that newly arrived sensory information will be encoded at a different field site in $u_{P E R}$, even if that information is repeated during the course of the sequence. The Past sequence memory field, $u_{P A}$ receives weak excitatory input from $u_{M E M}$ and as a result a memory trace of successive sequence demonstrations in $u_{P A}$ builds up. The functional role of this memory trace is to preshape neural populations in $u_{P E R}$ creating an expectation about upcoming perceptual events. This significantly speeds up the processing of the external input, which may be noisy and ambiguous, and is essential to adjust the sequence memory in successive demonstrations (for details see [11]).

As the result of the perceptual learning process, $u_{M E M}$ stores all sequence elements with the strength of activation decreasing from element to element as a function of elapsed

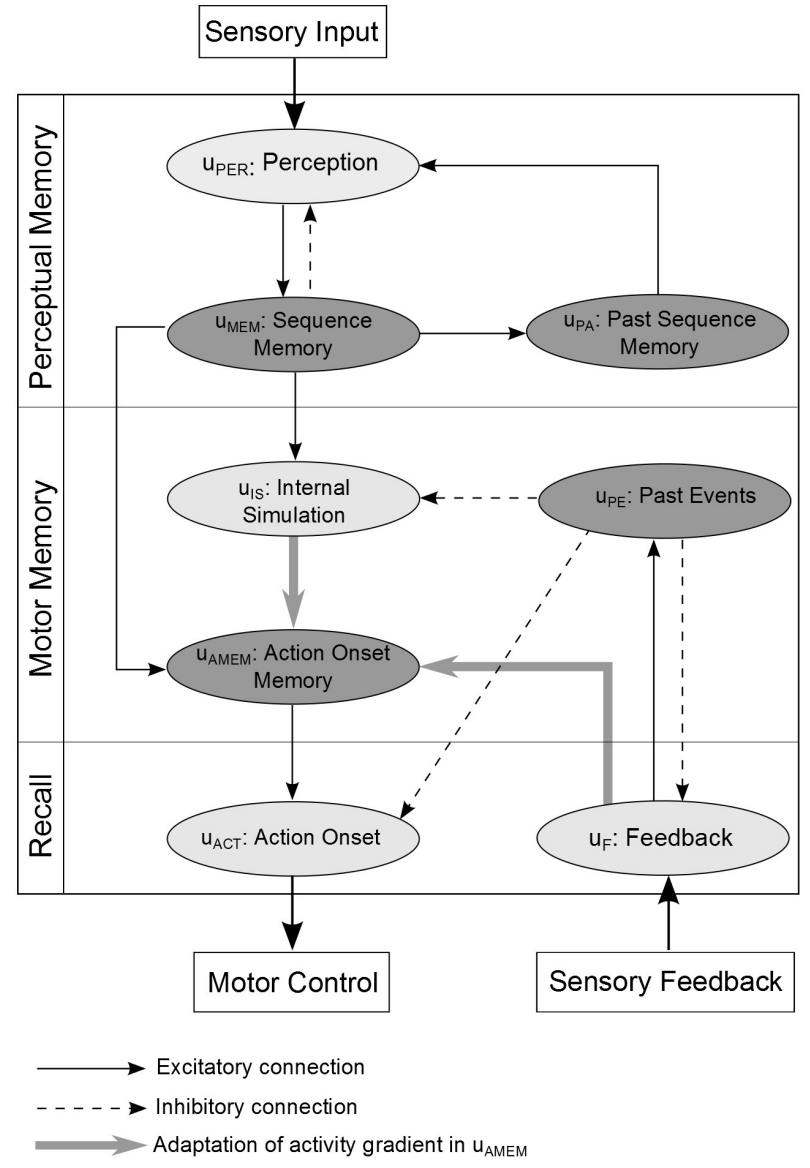

Fig. 1. Schematic view of the DNF architecture with several interconnected fields implementing perceptual sequence memory, motor sequence memory and sequence recall.

time since sequence onset. Fig. 2 shows an example of a sequence memory with 5 perceptual events (top panel). The stored information of order and timing can be overtly (in the robotics experiment) or covertly (in the simulation layer) recalled as shown by the time course of neural population activity encoding the different events (bottom panel). Note that the onset time of the suprathreshold population responses is aligned with the timing of the corresponding external events (vertical bars).

The perceptual memory represents abstract, effectorindependent sequence information. During sequence execution, the movement onset time has to be adjusted to compensate for the duration of the effector movements that are supposed to achieve the desired action effect at the expected time. Starting with the bias from the already acquired sequence knowledge, the dynamic interactions of neural populations in the motor memory and recall layers in a series of adaptation trials realize this goal. At the beginning of the first execution trial, the Action Onset Memory $\left(u_{A M E M}\right)$ equals the stored pattern in $u_{M E M}$, that is, the multi-bump in $u_{A M E M}$ represents the serial order and the expected relative timing of events. In the Internal Simulation Field $\left(u_{I S}\right)$, a covert recall of the perceptual memory $\left(u_{M E M}\right)$ is performed, whereas the Action Onset 

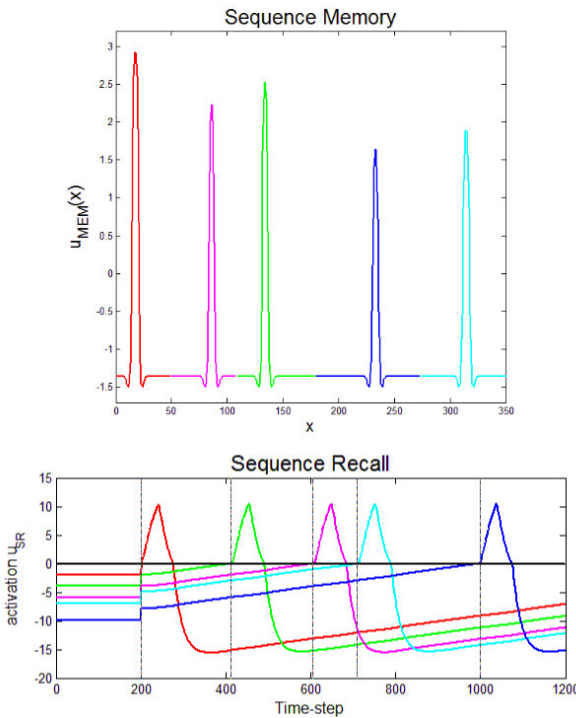

Fig. 2. Self-stabilized multi-bump pattern representing sequence memory (top). Time course of population activity during sequence recall (bottom). Vertical lines indicate the timing of the external events.

Field $\left(u_{A C T}\right)$ encodes overt sequence planning. The two fields receive a multi-bump pattern from $u_{M E M}$ and from $u_{A M E M}$, respectively, as a subthreshold input. Continuous increase of baseline activity in the preshaped fields $u_{I S}$ and $u_{A C T}$ brings all neural populations closer to the threshold necessary to trigger the evolution of a self-stabilized bump. When the most active population in $u_{A C T}$ reaches this threshold, the corresponding motor response (i.e. effector movement) is initiated. During movement execution, sensory or proprioceptive feedback is registered and used as an input to the Feedback Field $\left(u_{F}\right)$ which represents this input as a bump. The process of adaptation of the initial memory representation is based on a comparison between the time course of suprathreshold activity in $u_{F}$ and $u_{I S}$ to detect a potential mismatch between the expected timing and the currently executed timing of events. To compensate this mismatch, we adopt a simple learning rule proposed in [1] (see the pseudo-code below) to adapt the resting level in $u_{A M E M}$ as a function of the activity states in $u_{F}$ and $u_{I S}$.

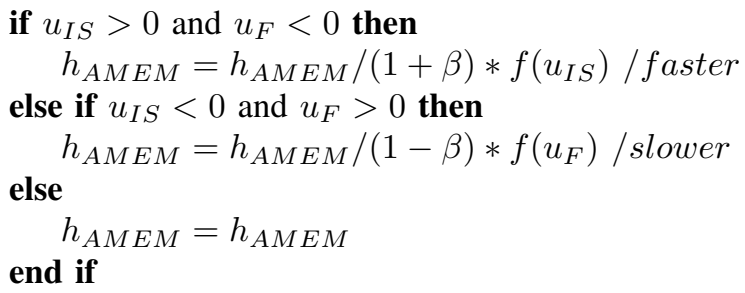

The adaptation of the resting level $h$ can be performed globally, that is, the $h$ value is changed for the whole field, leading to an equal temporal adjustments for all event representations, or locally at field sites with suprathreshold activity, leading to temporal adjustment of the currently processed action only. When the movement is executed with a delay, i.e. the suprathreshold activity appears later in $u_{F}$ than in $u_{I S}$, the resting level in $u_{A M E M}$ is increased in order to start the movement earlier in subsequent trials. Conversely, when the activity appears in $u_{F}$ earlier than in $u_{I S}$, the resting level in $U_{A M E M}$ is reduced to delay movement onset. During execution, the working memory of a realized action/event effect is stored in the Past Events field $\left(u_{P E}\right)$. Inhibitory connections to $u_{I S}, u_{A C T}$ and $u_{F}$ guarantee that existing bumps at corresponding field sites become suppressed. Once the activity is below threshold, the next action onset can be planned in $u_{A C T}$.

\section{Model EQuations}

As stated earlier, the model is based on the theoretical framework of Dynamic Neural Fields, that was originally proposed by Wilson and Cowan (1973) and Amari (1977) to explain pattern formation in a neural tissue [2], [23].

The population dynamics in each field is governed by a integro-differential equation, which describes the activation of a single layer of interconnected neurons along a onedimensional domain:

$\tau \dot{u}(x, t)=-u(x, t)+S(x, t)+\int w(x-y) f(u(y, t)) d y-h(t)$,

where $u(x, t)$ represents the activity at time $t$ of a neuron encoding feature value $x, \tau$ defines the time scale of the field dynamics, $S(x, t)$ represents the time dependent localized input at site $x$ from vision system and/or connected fields, $h(t)$ is the resting level to which the field activity converges without external stimuli, the coupling function $w(x-y)$ determines the strength of connectivity between neurons and $f(u)$ gives the firing rate function.

To implement a working memory function, the field dynamics must be in a bi-stable regime, that is, a stable homogeneous resting state representing the absence of information coexists with localized activation bumps representing specific value of the dimension represented by the field. In this regime, localized transient input of sufficient strength destabilize the resting state and drives the evolution of bump states whereas transient inhibitory input may be used to implement a "forgetting" mechanism. A continuous decrease of the global inhibitory input $h$ will systematically reduce the gap to the threshold for a bump formation of populations that get only subthreshold excitation from connected neuronal pools. Eventually, the excitation will be sufficiently strong to trigger a suprathreshold response. The interested reader may consult [2], [8] for a rigorous mathematical analysis of the memory function provided by the field dynamics in the bi-stable regime.

In his original work, Amari [2] analysed coupling function of lateral-inhibition type that does not support stable multibump solutions necessary to implement a memory functions for sequences. Laing et al. (2002) proposed a coupling function with oscillatory rather than monotonic decay and have shown that multiple regions of suprathreshold activity can persist simultaneously in a field. For the memory fields 
$u_{M E M}, u_{P A}, u_{P E}$ and $u_{A M E M}$ we adapt the connection function used in [14]:

$$
w(x)=A^{-b|x|}(b \sin |\alpha x|+\cos (\alpha x)),
$$

where the parameter $b>0$ controls the rate at which the oscillations in $w$ decay with distance. The parameters $A$ and $\alpha$ are added to control the amplitude and the zero crossings of $w$.

In the remaining fields, $u_{P E R}, u_{I S}, u_{A C T}$ and $u_{F}$, we apply a connection function of lateral inhibition type to guarantee the existence and stability of a 1-bump solution:

$$
w(x-y)=w_{e x c} e^{\left(\frac{(x-y)^{2}}{2 \sigma_{e x c}^{2}}\right)}-w_{i n h i b},
$$

where $w_{e x c}>0$ and $\sigma_{e x c}>0$ define, respectively, the amplitude and standard deviation, and $w_{i n h i b}>0$ represents a constant lateral inhibition.

The strength of individual actions representations in $u_{M E M}$ is controlled by a state-dependent dynamics for the baseline activity $h_{M E M}(x, t)[5]$ :

$$
\begin{aligned}
\dot{h}_{M E M}(x, t)= & \left(1-f\left(u_{M E M}(x, t)\right)\right)\left(-h_{M E M}(x, t)+h_{M E M_{0}}\right) \\
& +\frac{1}{\tau_{h_{M E M}}} f\left(u_{M E M}(x, t)\right),
\end{aligned}
$$

where $f$ is the Heaviside step function, $h_{M E M_{0}}<0$ defines the level to which $h_{M E M}$ converges without suprathreshold activity at position $x$ and $\tau_{h_{M E M}}>0$ measures the growth rate when it is present.

To retrieve the activation gradient from the memory, we apply a simple linear dynamics for the baseline activity of the $u_{I S}$ field:

$$
\tau_{h_{I S}} \dot{h}_{I S}(t)=1, \quad h_{I S}\left(t_{0}\right)=h_{I S_{0}}<0 .
$$

Changing the value of $\tau_{h_{I S}}$ allows for recalling the sequence with different speeds, while preserving the relative timing of events. However, to ensure that the sequence of events is executed with the same speed in $u_{I S}$ and $u_{A C T}$, time scale parameters must have the same value in both fields.

The adaptation of activation gradient in $u_{A M E M}$ is performed using the following $h$-dynamics:

$$
\begin{aligned}
\dot{h}_{A M E M}(x, t)= & \beta\left(1-f\left(u_{I S}(x, t)\right) f\left(u_{F}(x, t)\right)\right) \\
& \left(f\left(u_{F}(x, t)\right)-f\left(u_{I S}(x, t)\right)\right),
\end{aligned}
$$

where $f$ is again the Heaviside step function and $\beta$ is the adaptation rate parameter.

\section{RESULTS}

We report results of a model simulation that shall illustrate the basic functioning of the learning and adaptation of the joint representation of order and relative timing of perceptualmotor sequences. Fig. 3 shows a snapshot at time $t=700$ of the first execution trial of a perceptual sequence memory of 6 elements.

As can be seen in the figure, the subpopulation representing the fourth sequence element is above threshold in $u_{I S}$ and $u_{A C T}$, which means that at that moment the specific action is being executed. Neural populations in $u_{I S}$ and $u_{A C T}$
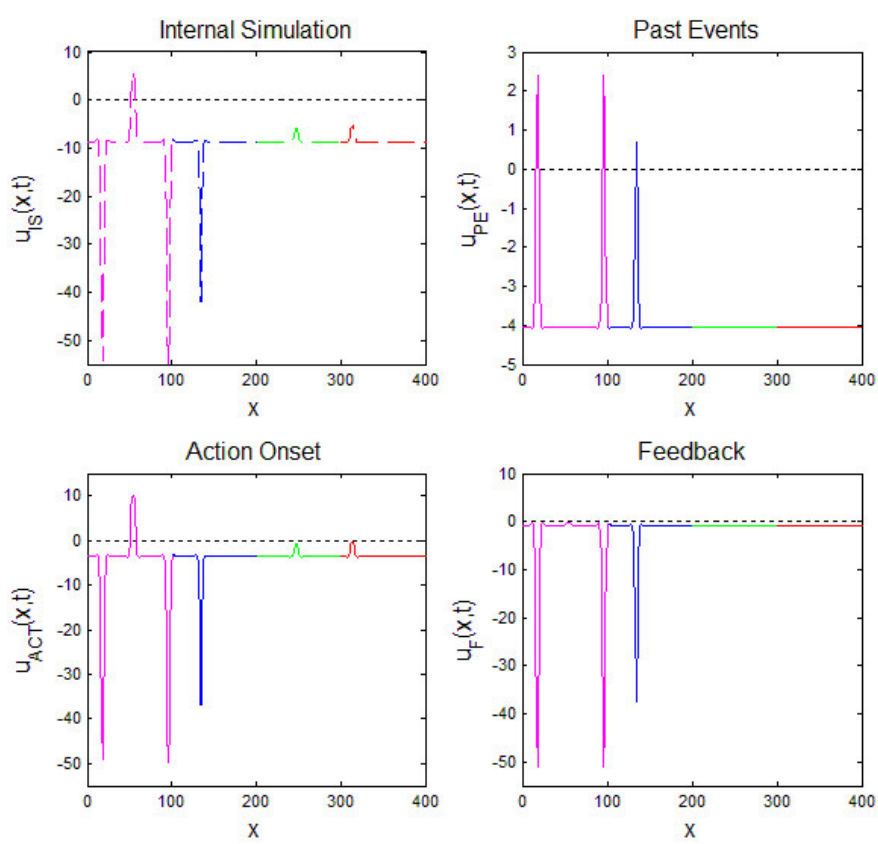

Fig. 3. Snapshot of field activities of a model simulation at time $t=700$.

appear to be preshaped by the tonic input from the memory field $u_{M E M}$, as can be seen by subthreshold activations of component actions that have not been executed yet. The activation peak in $u_{F}$ is just about to reach the threshold since the processing of feedback is delayed with respect to the expected timing represented in $u_{A C T}$. Once the subpopulation in $u_{F}$ becomes active, neurons in $u_{F}$ drive through excitatory connections the evolution of a suprathreshold activity peak in the connected population in $u_{P E}$. Mediated by inhibitory connections, the existence of the peak in $u_{P E}$ automatically suppresses the suprathreshold activity in the corresponding populations in $u_{I S}, u_{F}$ and $u_{A C T}$. This negative feedback can be clearly seen at the field locations of the three action representations that have been already executed.

Details of the activity gradient adaptation in $u_{A M E M}$ are discussed next.

\section{A. Effect of a global adaptation of the resting level in $u_{A M E M}$}

At the beginning of the first execution trial, the activity gradients in $u_{M E M}$ and in $u_{A M E M}$ are equal. Assuming equal time scales for the $h$-adaptation in $u_{I S}$ and $u_{A C T}$, the simulated timing of the expected action effect matches the executed onset of the first movement. Obviously, the sensory feedback about the execution of the first action appears to be significantly delayed and consequently also the execution of the whole sequence. To change sequence onset to earlier times, a global $h$-level adaptation has been used, as shown in Fig. 4. This increases the gradient as a whole without affecting the relative timing encoded in the relative strengths of population representations of successive actions (top). The resulting stronger preshaping in $u_{A C T}$ reduces in subsequent learning trials the movement onset for all component actions 

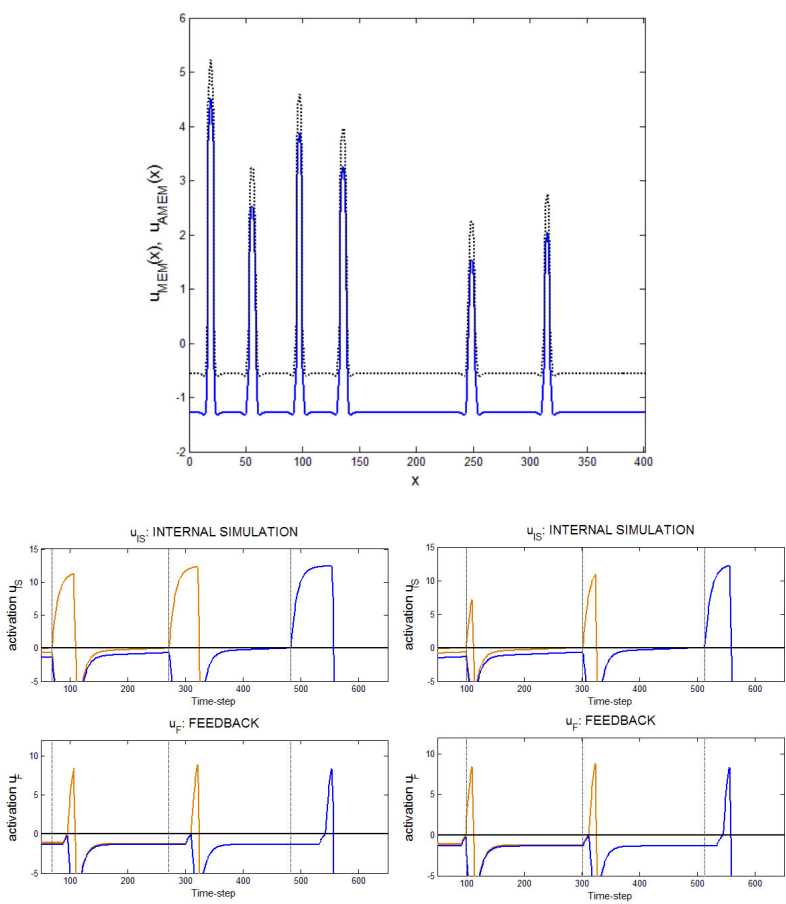

Fig. 4. Global adaptation of resting level in $u_{A M E M}$. Top panel of the figure presents comparison of Perceptual Sequence Memory $u_{M E M}$ (solid line) and Action Onset Memory $u_{A M E M}$ (dotted line). Bottom panel shows the time course of activity in $u_{I S}$ and $u_{F}$ before (left) and after (right) the global h-level adaptation.

by a constant amount of time (bottom).

The change in the resting level is governed by equation (6), with an adaptation parameter $\beta$ which decreases over learning iterations $i$ :

$$
\beta=\lambda_{1} e^{-i}+\lambda_{2}
$$

where $\lambda_{1}$ and $\lambda_{2}$ are constant.

This increases the efficiency of the adaptation process since the temporal difference between the time course of suprathreshold activations in $u_{I S}$ and $u_{F}$ can be initially quite big, allowing a larger adaptation of the resting state per iteration. Smaller values of $\beta$ on the other hand guarantee fine adjustment when the expected timing of events and the time of sensory feedback are already relatively close.

For the case of constant delays for all component actions, like for the keystrokes in the piano task [11], this global $h$ adaptation is sufficient to perfectly synchronize movement onset with external events (e.g., a metronome). However, assuming different movement times for individual events, we still need a mechanism to adjust the amplitudes of particular peaks of activation in $u_{A M E M}$. This can be seen when comparing the time courses of the populations representing actions 2 and 3 in Fig. 4 (bottom) which are still delayed.

\section{B. Effect of a local adaptation of the resting level in $u_{A M E M}$}

To fine tune the timing of each particular event represented in the initial perceptual memory, we used a local $h$-adaptation in accordance with equation 6. In the example shown in

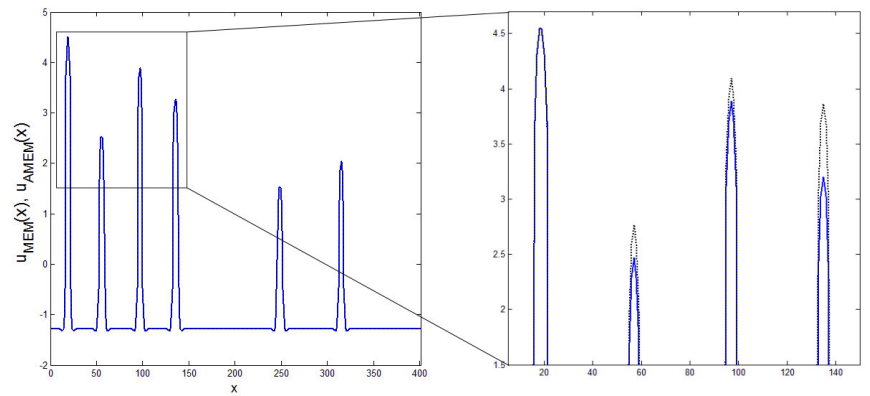

Fig. 5. Result of local adaptation of resting level in $u_{A M E M}$. Left panel: Perceptual Sequence Memory $u_{M E M}$ (solid line) and Action Onset Memory $u_{A M E M}$ (dotted line) are compared. Right panel: To make changes in the peak amplitudes better visible, the amplitudes have been multiplied by a constant factor.

Fig. 5, all action onsets except the first one are delayed by varying amounts of time. The local adaptation mechanism has the objective to minimize the difference in the onset of suprathreshold activity of corresponding local populations in $u_{I S}$ and $u_{A C T}$. The resulting increase in peak amplitudes in $u_{A M E M}$ compared to $u_{M E M}$ indicates that movement onset times appear to be reduced. To verify that the novel motor memory indeed represents the correct timing of events, Fig. 6 compares for two action representations the time courses before and after the adaptation process. The bottom panel shows that the earlier movement onsets produce the action effects at the time predicted by the internal simulation.

Since the success of the local adaptation process does not depend on the magnitude of the initial temporal mismatch, it becomes clear that a global $h$-adaptation is not a necessary first step. The adjustment of the timing could be achieved by the local process alone. However, performing the global adaptation first significantly accelerates the adaptation process, reducing the number of execution-adaptation trials needed to achieve a satisfactory result. The speed of convergence of the process depends of course also on the choice of the adaptation rate parameter $(\beta)$. As can be seen in the Fig. 7, larger values of $\beta$ lead as expected to a faster convergence. However, increasing $\beta$ beyond a certain limit may introduce instabilities in the adaptation process. With larger changes in the memory strengths in each iteration, the temporal differences between the onset of suprathreshold activity in $u_{I S}$ and $u_{F}$ start to oscillate between positive and negative values.

\section{DiscusSiON}

In this paper, we have used the neuro-plausible modeling framework of dynamic fields to address the problem of temporal and ordinal learning of perceptual-motor sequences. The simulation results demonstrate how feedback about executed actions might be used by the cognitive system to fine tune a joint memory representation of the ordinal and the temporal structure which has been initially acquired by observational learning. The fluent succession of acts in our performance of highly-trained, sequential behaviors is generally believed to support the notion of joint representations [12], [17]. However, the need to rapidly adapt the movement 

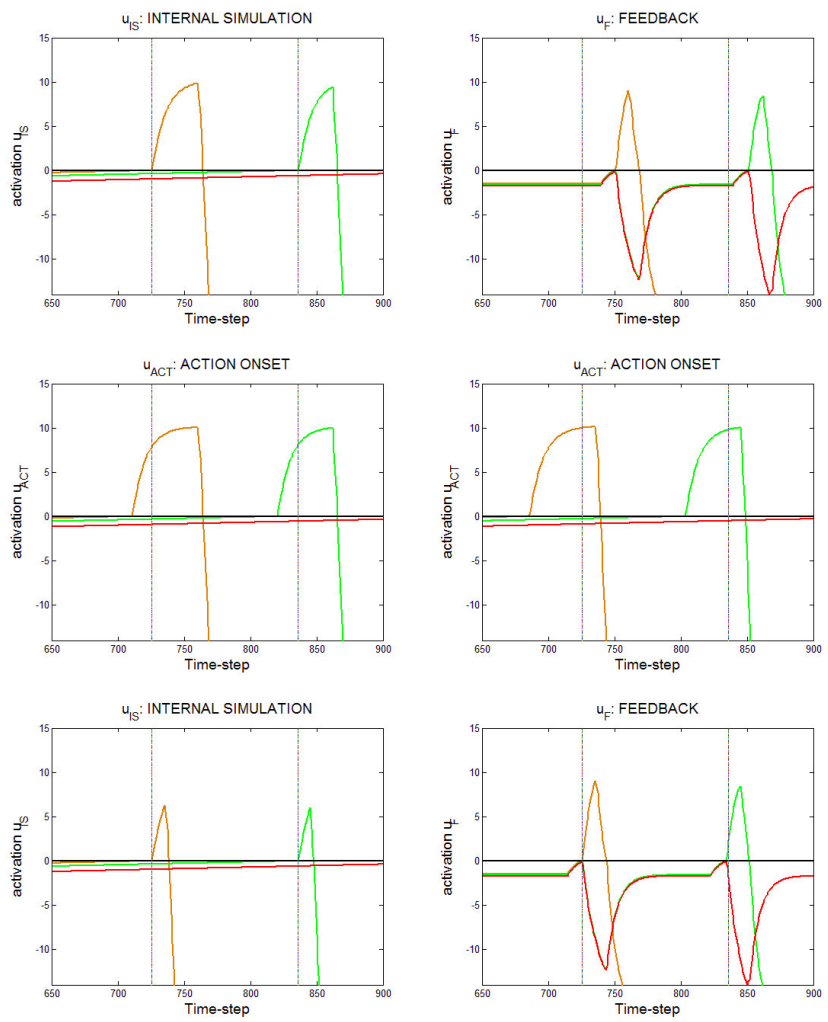

Fig. 6. Effect of a resting level adaptation in $u_{A M E M}$ on the timing of events. The top panel of the figure presents the time course of population activity representing two successive sequence elements in $u_{I S}$ (left) and $u_{F}$ (right) after the first execution of the sequence. The middle panel shows the time course of activity in $u_{A C T}$ before (left) and after (right) the local hlevel adaptation. The bottom part of the figure presents the time course of activity in $u_{I S}$ (left) and $u_{F}$ (right) after the adaptation process in $u_{A M E M}$
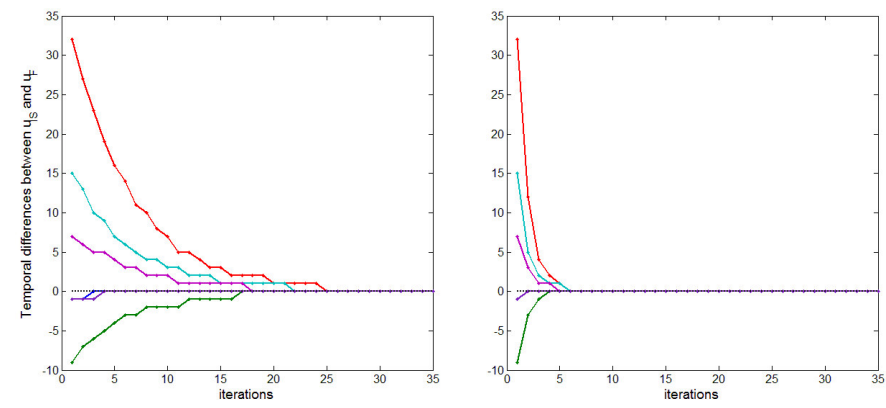

Fig. 7. The change in the delay between the onset of suprathreshold activity in $u_{I S}$ and $u_{F}$ with increasing numbers of iterations is plotted for $\beta=$ 0.0005 (left) and $\beta=0.002$ (right).

rates to changing environmental constraints has been used as argument against a fully integrated view [21]. The model architecture solves this problem by assuming that a joint memory representation is integrated in the action onset layer with a separate action onset mechanism which preserves the relative inter-response times encoded in the memory.

Conceptually, the model belongs to a class of theoretical approaches known as competitive cuing (CQ) models that have been widely used in the past to explain data from human sequence learning ([13], for review and discussion see [18]).
The parallel planning of all sequence elements is one of the essential features that distinguishes CQ-model from the fundamentally serial mechanism implemented in also very popular recurrent neural network models of serial organization (e.g., [6], [7]). The DNF model extends the CQ-principle of storing serial order in an activation gradient to include also temporal information. The action onset mechanism used to recover the stored action timing is consistent with neuroplausible models of decision making. These models assume that a decision signal rises in response to information about the task at hand until a fixed decision threshold is reached (e.g., [20]). In the action onset layer, neural population activity rises linearly from a pre-activated state due to the release of global inhibition. The recent concept of proactive inhibitory control that has been discussed in relation to movement preparation and reaction time is in line with the implemented task-dependent adjustment of the baseline activity of neural populations [22]. It is important to notice that the simple linear dynamics implemented in the model is not a prerequisite, other growth models could have been used as well as long as the growth rate matches the growth rate of the dynamics controlling the strength of the memory representations.

While the presented modeling work takes inspiration from neurophysiological and behavioral findings, it is also constrained by the specific needs of robotics applications. Our long-time goal is to develop autonomous robots able collaborate with humans in a natural that is human-like manner [9]. We have tested in the past a complex cognitive control architecture consisting of several coupled DNFs in a task in which the robot ARoS collaborates with different users in assembling a toy object from its parts [3]. As shown in many real world robotics applications [8], the processing principle of recurrent excitatory feedback from neighboring neurons has been proven efficient in amplifying noisy and often weak sensory signals. Moreover, the self-stabilizing properties of the field dynamics can be exploited to implement a robust decision making capacity in ambiguous and noisy environments. In the present study, we have used the formation of stable bumps to test an activation-based learning mechanism that supports the rapid acquisition of sequential knowledge by observation. Observational learning is considered highly attractive for robotics in general since it may significantly speed up skill acquisition compared to individual discovery in potentially dangerous trial-and-error learning [4]. Since many demonstration would annoy the teacher, the learning should be fast and efficient. In the observational learning study mentioned in the introduction, ARoS was able to correct initial memory errors in sequencing and timing of a musical sequence consisting of 12 sensory events in only 4-5 demonstration-execution cycles. Due to noise in the encoding and the recall phase, the effective timing could vary from trial to trial but still in a range that preserved a recognizable melody.

The modeling results show that the perceptual memory of observed sequential information may be used by the cognitive system as a bias to efficiently develop an effector-specific 
motor memory of serial performance. The adaptation rule implements a comparison between the dynamic states of neuronal populations in different model layers representing the expected and realized timing of behavior. The mechanisms is similar to the action monitoring function implemented in our DNF architecture for human-robot interactions [3].

The focus of the present theoretical work on the important temporal dimension of sequential behavior does not mean that all aspects of the order problem have been already solved. For instance, the order of component actions may not be fixed but may vary depending on the task context. Extracting common principles from multiple examples is the hallmark of recurrent neural network models [6], [7]. From the robotics point of view, it would be interesting to combine the advantage of a fast activation-based learning by observation with a slower weight-based learning to extract such generalized task knowledge [16].

Directly testing in the piano task the feedback learning extension of the original DNF model was not possible due to limitations in the precision of the arm and hand control. We are currently exploring the learning of goal-directed action sequences such as reaching-grasping-transporting-placing an object that can be segmented in a series of contact events. Compared to the musical example, a much reduced temporal precision is needed (in the range of several seconds not milliseconds) to produce useful results. Learning and flexible adapting the timing of these sequences will greatly improve the smoothness and skillfulness of human-robot interactions.

\section{ACKNOWLEDGMENT}

This research was supported by the Marie Curie Network for Initial Training NETT and the FCT-Research Center CMAT (PEst-OE/MAT/UI0013/2014).

\section{REFERENCES}

[1] R. Almeida and A. Ledberg. A biologically plausible model of timescale invariant interval timing. Journal of computational neuroscience, 28(1):155-175, February 2010.

[2] S. Amari. Dynamics of pattern formation in lateral-inhibition type neural fields. Biological Cybernetics, 27(2):77-87, 1977.

[3] E. Bicho, W. Erlhagen, L. Louro, and E. Costa e Silva. Neuro-cognitive mechanisms of decision making in joint action: A humanrobot interaction study. Human Movement Science, 30(5):846 - 868, 2011.

[4] A. Billard, S. Calinon, R. Dillmann, and S. Schaal. Robot programming by demonstration. In Bruno Siciliano and Oussama Khatib, editors, Springer Handbook of Robotics, pages 1371-1394. Springer Berlin Heidelberg, 2008.

[5] S. Coombes and M. Owen. Exotic dynamics in a firing rate model of neural tissue with threshold accommodation. AMS Contemporary Mathematics, 44:123-144, February 2007.
[6] P. F. Dominey. A shared system for learning serial and temporal structure of sensori-motor sequences? evidence from simulation and human experiments. Cognitive Brain Research, 6(3):163 - 172, 1998.

[7] J. L. Elman. Finding structure in time. Cognitive Science, 14(2):179$211,1990$.

[8] W. Erlhagen and E. Bicho. The dynamic neural field approach to cognitive robotics. Journal of Neural Engineering, 3:36-54, 2006.

[9] W. Erlhagen and E. Bicho. A dynamic field approach to natural and efficient human-robot collaboration. In beim Graben P. Pothast R. Weight. J. Coombes, S., editor, Neural Fields: Theory and Applications, pages 341-365. Springer Berlin Heidelberg, 2014.

[10] F. Ferreira, W. Erlhagen, and E. Bicho. A dynamic field model of ordinal and timing properties of sequential events. In Timo Honkela, Wlodzislaw Duch, Mark A. Girolami, and Samuel Kaski, editors, ICANN (2), volume 6792 of Lecture Notes in Computer Science, pages 325-332. Springer, 2011.

[11] F. Ferreira, W. Erlhagen, E. Sousa, L. Louro, and E. Bicho. Learning a musical sequence by observation: A robotics implementation of a dynamic neural field model. In Development and Learning and Epigenetic Robotics (ICDL-Epirob), 2014 Joint IEEE International Conferences on, pages 157-162, Oct 2014.

[12] E. W. Gobel, D. J. Sanchez, and P. J. Reber. Integration of temporal and ordinal information during serial interception sequence learning. Journal of Experimental Psychology: Learning, Memory, and Cognition, 2011.

[13] S. Grossberg. Behavioral contrast in short term memory: Serial binary memory models or parallel continuous memory models? Journal of Mathematical Psychology, 17(3):199 - 219, 1978.

[14] B. S. Gutkin, C. R. Laing, B. Ermentrout, and W. C. Troy. Multiple bumps in a neuronal model of working memory. SIAM Journal of Applied Mathematics, 63(1):62-97, 2002.

[15] K. Kornysheva, A. Sierk, and J. Diedrichsen. Interaction of temporal and ordinal representations in movement sequences. Journal of Neurophysiology, 109(5):1416-1424, 2013.

[16] J. L. McClelland, B. L. McNaughton, and R. C. O'Reilly. Why there are complementary learning systems in the hippocampus and neocortex: Insights from the successes and failures of connectionist models of learning and memory. Psychological Review, 102:419-457, 1995.

[17] J. X. O'Reilly, K. J. McCarthy, M. Capizzi, and A. C. Nobre. Acquisition of the Temporal and Ordinal Structure of Movement Sequences in Incidental Learning. J Neurophysiol, 99(5):2731-2735, May 2008.

[18] B. J. Rhodes, D. Bullock, W. B. Verwey, B. B. Averbeck, and M. P. A Page. Learning and production of movement sequences: Behavioral, neurophysiological, and modeling perspectives. Human Movement Science, 23(5):699-746, November 2004.

[19] R. I. Schubotz, A. D. Friederici, and D. Y. von Cramon. Time perception and motor timing: a common cortical and subcortical basis revealed by fMRI. Neuroimage, 11(1):1-12, January 2000.

[20] D. Standage, H. You, Da-Hui H. Wang, and M. C. Dorris. Gain modulation by an urgency signal controls the speed-accuracy tradeoff in a network model of a cortical decision circuit. Frontiers in computational neuroscience, 5, 2011.

[21] F. Ullén and S. L. Bengtsson. Independent processing of the temporal and ordinal structure of movement sequences. Journal of Neurophysiology, 90(6):3725-3735, 2003.

[22] C. Wardak, S. Ramanol, O. Guipponi, P. Boulinguez, and S. B. Ben Hamed. Proactive inhibitory control varies with task context. European Journal of Neuroscience, 36(11):3568-3579, 2012.

[23] H. R. Wilson and Jack D. Cowan. A mathematical theory of the functional dynamics of cortical and thalamic nervous tissue. Kybernetik, 13:55-80, 1973 